\title{
Alternating spin-polarized current induces parametric resonance in spin valves
}

\author{
Marcel G. Clerc, ${ }^{1}$ Saliya Coulibaly, ${ }^{2}$ David Laroze, ${ }^{3,4}$ Alejandro O. León, ${ }^{1}$ and Álvaro S. Núñez ${ }^{1}$ \\ ${ }^{1}$ Departamento de Física, Facultad de Ciencias Físicas y Matemáticas, Universidad de Chile, Casilla 487-3, Santiago, Chile \\ ${ }^{2}$ Laboratoire de Physique des Lasers, Atomes et Molécules, Centre National de la Recherche Scientifique, Unités Mixtes de Recherche 8523, \\ Université des Sciences et Technologies de Lille, 59655 Villeneuve d'Ascq Cedex, France \\ ${ }^{3}$ Instituto de Alta de Investigación, Universidad de Tarapacá, Casilla 7D, Arica, Chile \\ ${ }^{4}$ SUPA School of Physics and Astronomy, University of Glasgow, Glasgow G12 8QQ, Scotland, United Kingdom
}

(Received 19 January 2014; revised manuscript received 7 June 2015; published 23 June 2015)

\begin{abstract}
Ferromagnetic systems under the influence of spin-polarized currents exhibit rich spatiotemporal dynamics at nanoscales. We study spin-transfer nano-oscillators driven by the combination of alternating and direct spin-polarized electric currents. We show here that the alternating current induces parametric instabilities on spin valves, that is, the magnetization responses at half the forcing frequency. A spatial self-organization emerges as a result of the oscillatory current, which includes dissipative solitons and Faraday-type waves. The parametric regime is described analytically by means of the Landau-Lifshitz-Gilbert-Slonczewski equation, in good agreement with micromagnetic simulations including the full dipolar field.
\end{abstract}

DOI: 10.1103/PhysRevB.91.224426

PACS number(s): 75.78.Fg, 85.75.-d, 89.75.Kd

\section{INTRODUCTION}

Control of magnetization in spin valves has been the subject of intense research in recent years [1-4]. In such devices, a perpendicular to plane electric current transfers spin angular momentum from one ferromagnetic film into another one. This effect is known as the spin-transfer torque $[1,5,6]$, and it can generate magnetic switching or reversals, and stable oscillatory states in the radio-frequency domain. Furthermore, the addition of an alternating electric current, in the radio-frequency domain, affects the reversal threshold through a frequency locking mechanism. The injection of combined radio-frequency and direct electric currents induces other dynamical responses, such as synchronization, chaos [7], and stochastic resonance [8].

Although the first predictions on current-induced dynamics were made assuming a uniform magnetization (the macrospin model), it is known that usually both switching and precessional motions are nonuniform $[9,10]$. Moreover, a direct spinpolarized electric current can induce static spatially periodic textures in magnetic films [11,12]. Vortices are a classical example of localized magnetization states [13]. In general, vortex stability is related to the geometrical properties of the sample [14]. A spatial distribution of the applied current can also modify the magnetic states. For example, the case of metallic point contact, in which the current is applied through a small metallic cross section in contact with a continuous magnetic film, exhibits nonuniform magnetization states [15]. In addition, magnetic solitonic modes in nano-oscillators have been observed [16-18]. Recently, dissipative magnetic droplet solitons were experimentally found and studied [19], after they were theoretically predicted in Ref. [20].

The study of spatially self-organized structures is beyond the specific case of magnetization dynamics; moreover, it is a widespread topic in nonlinear science [21-30]. Among the large variety of patterns generated by out-of-equilibrium nonlinear systems, dissipative localized structures [21,22,29] have always been of great interest and extensively studied for their potential applications. In the context of spin transfer, localized states can be of great advantage, since the energy localization that they produce is independent of the geometrical properties of the system. Usually the existence of localized structures is related to a subcritical bifurcation [31]. This is, for example, the case of parametrically driven systems [29]. Parametric driving occurs when energy or momentum is injected in a system by means of a temporal modulation of one or more parameters. Hence, resonance can produce a response frequency different from the forcing frequency. The best known case is the 2:1 resonance phenomenon, where the driving frequency is close to twice the natural frequency of the system [32]. Parametric excitation of localized states arises in a wide range of physical systems. Examples include vertically oscillating layers of water, nonlinear lattices, optical fibers, Kerr-type optical parametric oscillators, the magnetization in an easyplane ferromagnet exposed to an oscillatory magnetic field, and a parametrically driven damped chain of pendula. In most cases parametric forcing is made by modulating one of those parameters on which the natural frequency of the system depends.

In the context of spin-transfer nano-oscillators, currentinduced parametric excitations were recently observed in point-contact spin-valve nanodevices [33,34]. Point contacts inject direct and microwave currents into spin valves, thus generating oscillating spin-transfer and Oersted-field torques on magnetic moments. Both effects contribute to the parametrically excited dynamics. This inhomogeneous forcing induces a fixed vortex. As a result of inhomogeneous forcing, the dynamics of these systems corresponds to nonextended parametric systems.

In this paper, we demonstrate theoretically and numerically that a uniform alternating spin-polarized electric current can produce parametric excitation in spin-valve devices. Figure 1 shows the typical dynamical response under parametric injection, in which the magnetization oscillates at half the forcing frequency. A parametrically induced resonance occurs when the self-oscillation induced by the direct current couples with the alternating current oscillation. A minimal model describing the dynamics of the magnetization at the onset of this resonance is given. We also show that such parametrically driven spin-transfer nano-oscillators (PDSTNOs) generate self-organized magnetic structures such as dissipative solitons and Faraday-type waves. To verify the robustness of such 


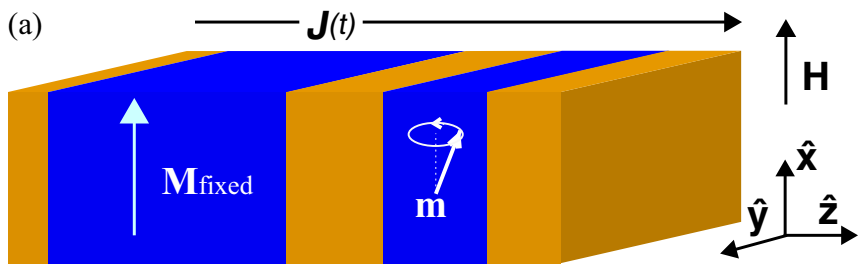

(b)
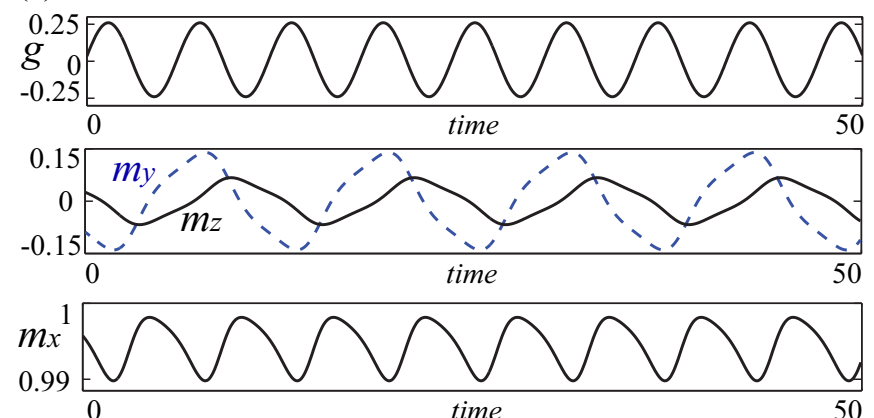

FIG. 1. (Color online) Schematic representation of the spintransfer nano-oscillator device and typical subharmonic response. (a) A multilayer nanopillar with free magnetization $\mathbf{m}$, and fixed magnetization along $\mathbf{M}_{\text {fixed }}$. (b) Parametrically induced oscillation obtained from Eq. (1), where $g$ is the dimensionless alternating current parameter; the dynamical variables $m_{y}$ and $m_{z}$ oscillate at half the frequency of the electric current, while the magnetization component $m_{x} \sim 1-\left(m_{y}^{2}+m_{z}^{2}\right) / 2$ precesses at the forcing frequency.

states, we conduct micromagnetic simulations including the full dipolar field.

The paper is organized as follows. In Sec. II we investigate the effect of a time-dependent spin-polarized current under the approximation of a uniform magnetization. In Sec. III we extend our study to nonuniform magnetization dynamics. Finally, in Sec. IV we give our main conclusions and remarks.

\section{PARAMETRICALLY DRIVEN SPIN-TRANSFER NANO-OSCILLATORS IN THE MACROSPIN APPROXIMATION}

Let us consider a spin-valve or nanopillar device, composed by two ferromagnetic layers separated by a nonmagnetic conductor as depicted by Fig. 1(a). One magnetic film has a fixed magnetization along the positive $\hat{x}$ axis, while the uniform magnetization of the second magnet $\mathbf{M}(t)$ is variable or free. The free magnetization obeys the dimensionless LandauLifshitz-Gilbert-Slonczewski (LLGS) equation [5,35,37]:

$$
\dot{\mathbf{m}}=-\mathbf{m} \times \mathbf{h}_{\mathrm{eff}}+\alpha \mathbf{m} \times \dot{\mathbf{m}}+g \mathbf{m} \times(\mathbf{m} \times \hat{x}),
$$

where $\mathbf{m}=\mathbf{M} / M_{s}$ is the normalized magnetization of the free layer, $M_{s}$ is its saturation magnetization, and $\dot{\mathbf{m}}$ stands for the temporal derivative of $\mathbf{m}$. Within the macrospin approximation, the vector $\mathbf{m}(t)$ is uniform. The time is rendered dimensionless using $M_{s}$ and the gyromagnetic constant $\gamma$, $t \rightarrow \gamma M_{s} t$. The first term of the right-hand side of Eq. (1) favors precessions around the effective field $\mathbf{h}_{\text {eff }}$ :

$$
\mathbf{h}_{\mathrm{eff}}=\left(h_{0}+\beta m_{x}\right) \hat{x}-m_{z} \hat{z},
$$

where $\beta$ stands for the easy axis coefficient (in this case the $x$ axis), and $h_{0}=H_{0} / M_{s}$ is the normalized external magnetic along the $x$ axis. The last term of the effective field is the demagnetization field, and it disfavors configurations along the $z$ axis. The second term of Eq. (1) accounts for the phenomenological Gilbert damping. The spin-transfer torque is modeled in Eq. (1) with the term proportional to $g$. The coefficient $g$ is defined by

$$
g=\frac{\hbar}{2|e| d} \frac{\mathcal{P} J}{\mu_{0} M_{s}^{2}},
$$

where $\mathcal{P}$ describes the electron polarization at the interface between the magnet and the spacer, $J$ is the current density, $|e|$ is the modulus of the electric charge, and $d$ is the thickness of the layer. In the spin-transfer theory of Slonczewski [5], the coefficient $g$ is a function of the magnetization, $g=$ $g(\mathbf{m} \cdot \hat{x})$. Let us focus on the small applied currents and small amplitude oscillations around the $\hat{x}$ axis; in this regime we can approximate $g(\mathbf{m} \cdot \hat{x}) \approx g(1)$; this approach is known as sine approximation [37].

In a linear regime the dynamics of the magnetization can be described by only one independent variable. Indeed, assuming $m_{y}^{2} \sim m_{z}^{2} \ll 1$, one obtains

$$
m_{z}=\frac{1}{1+h}\left(\frac{\alpha g}{1+h}-1\right)\left[\dot{m}_{y}+(\alpha h-g) m_{y}\right],
$$

and

$$
\ddot{m}_{y}=-\omega^{2} m_{y}-2 \tilde{\mu} \dot{m}_{y}+\dot{g} m_{y}+\frac{\alpha \dot{g}}{1+h} \dot{m}_{y},
$$

with $h=h_{0}+\beta, \omega^{2}=\left(1+\alpha^{2}\right)\left[h(1+h)+g^{2}\right]$, and $\tilde{\mu}=$ $\alpha(h+1 / 2)-g$. Thus, the magnetization satisfies the equation of an oscillator.

In the case of a direct current $\left(g=g_{0}\right)$, the stability analysis of Eq. (4) shows a stationary bifurcation at $(h+1 / 2)^{2}+g_{0}^{2}-(1 / 2)^{2}=0$, and an Andronov-Hopf bifurcation at $\alpha(h+1 / 2)-g_{0}=0[1,11]$. The Andronov-Hopf instability is responsible for the self-oscillations in spintransfer nano-oscillators. Let us consider a periodic timedependent current injection. In the linear approximation, Eq. (4) is a Mathieu-type equation with a damping force. Such a model is commonly solved by means of the classical Floquet method [38], which allows the determination of the instability regions-Arnold tongues - in parameter space. Hence, parametric resonance occurs in regions where the motion of the system is unstable with respect to driving frequencies that are different from its natural frequency. In what follows we will focus on the case where the driving frequency is close to twice the natural frequency of the system. In this case, a modal decomposition method [39] has been used to solve Mathieu-type equations. More precisely, if we assume a spin-polarized current of the form $g(t)=g_{0}+g_{1} \sin (\omega t)$, the parametric instability region can be obtained using the following trial function (Galerkin expansion [40]):

$$
m_{y}(t)=p_{1}^{0} e^{i \frac{\omega}{2} t}+p_{3}^{0} e^{i \frac{3 \omega}{2} t}+\text { c.c. },
$$

where the coefficients $p_{1,3}^{0}$ are constants and the symbol c.c. means complex conjugate. Introducing the ansatz (5) into Eq. (4), we find the following solvability condition for nonzero coefficients $p_{1}^{0}$ and $p_{3}^{0}$ :

$$
\left.|a| \Lambda\right|^{2}+\left.L g_{1}^{2}\right|^{2}-b^{2}|\Lambda|^{4} g_{1}^{2}=0,
$$



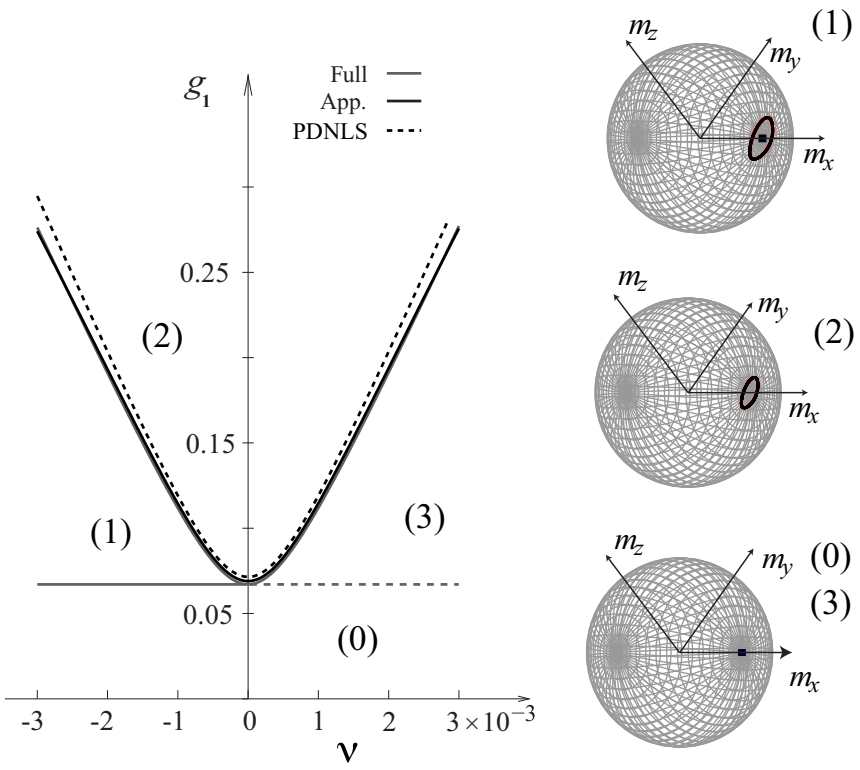

FIG. 2. (Color online) Bifurcation diagram (left) and phase portraits (right) of the parametric resonance in the macrospin limit. The parameters are $h_{0}=0.2, \beta=0.05, g_{0}=0.018, \alpha=0.025$, and $g_{1}=0.2$. The detuning parameters are $v=-0.005$ in region (1), -0.0005 in region (2), and 0.05 in region (3). The solid line (red curve) is given by the full equation using a trial function up to the fifth harmonic and can be considered as the exact limit in the quasireversible case. The solid black line is obtained from Eq. (6), while the black dashed line comes from the amplitude equation (8). The dashed red line gives the transition to the Faraday instability and may be taken into account only in the micromagnetic limit.

where $\quad L=\left(|c|^{2}-i \operatorname{Re}(c) b-b^{2}\right) \bar{\Lambda}-\operatorname{Re}(\Lambda) a-|\Lambda|^{2} / 2$. Here we have set $\omega_{0}^{2} \equiv\left(1+\alpha^{2}\right)\left[h(h+1)+g_{0}^{2}\right], a \simeq 2 \omega_{0}$ $(v-i \mu), b \simeq g_{0}-\alpha \omega_{0}^{2} /(1+h), c \simeq-2 \omega_{0}\left[1-i \alpha \omega_{0} /(1+h)\right]$, $\delta \simeq 4 \omega_{0}\left(2 \omega_{0}-i \mu\right), \quad \mu \equiv \alpha\left(h+\frac{1}{2}\right)-g_{0}, \quad \Lambda=a+\delta$, and $v=\omega / 2-\omega_{0}$ represents the detuning parameter. Notice that in Eq. (6) we have considered only terms up to the order of $\mu_{0} \sim g_{0} \sim g_{1}^{2} \sim v \sim \alpha$ and $\alpha \ll 1$. The relationship (6) is depicted by the black solid line in Fig. 2. We have compared this curve with that given by the full numerical solution obtained with a trial function up to the fifth harmonic (gray line). These two curves are almost indistinguishable in this figure. Hence, our reduced approximation provides an excellent description of the boundaries of the parametric instability. The region inside the curve accounts for the Arnold tongue [41]. In this region, the steady magnetization state, $\mathbf{m}=\hat{\mathbf{x}}$, undergoes an instability, which is saturated in a stable precessional state corresponding to a limit cycle in phase space. Figure 1(b) shows the typical temporal evolution of the magnetization components inside the Arnold tongue. The right panel in Fig. 2 illustrates the precessional and uniform states in the magnetization space. Outside this tongue, in region (1) outlined in the left panel of Fig. 2, the precessional state persists and coexists with the stable uniform magnetization. The precessional state exhibits a saddle-node bifurcation when going from region (0) to region (1).

The dynamics of parametrically forced systems can be decomposed into fast and slow temporal scales at the onset of their subharmonic resonance [21]. The fast scale is given by the oscillation frequency $\omega_{0} \approx \omega / 2$, while the slow scale is the evolution of the oscillation envelope. The description of the PDSTNO at the onset of resonance, in terms of the oscillation amplitude, is obtained by introducing the following ansatz:

$$
m_{y}=m_{y, 0}\left[A e^{i\left(\omega_{0}+v\right) t}+\bar{A} e^{-i\left(\omega_{0}+v\right) t}\right]+W_{y},
$$

where the normalized oscillation envelope $A$ describes the magnetization deviation from the homogeneous orientation $\mathbf{m}=\hat{\mathbf{x}}$. The normalization constant $m_{y, 0}=$ $-\sqrt{2 \omega_{0} /\left(3 N_{1}+\omega_{0}^{2} N_{2}\right)}$, where $N_{1} \simeq[(1+2 \beta) h+\beta] / 2$, and $N_{2} \simeq(1+\beta)(h-2) /\left[2(h+1)^{2}\right]+\beta /[2(h+1)]$ describes the characteristic scale of oscillations. $W_{y}$ is a higher-order correction that depends nonlinearly on $A$ and its complex conjugate. Introducing formula (7) in Eq. (1), linearizing $W_{y}$ and applying the solvability condition, we get the parametrically driven and damped nonlinear Schrödinger equation (PDNLS) without space $[21,29]$ :

$$
\dot{A}=-i v A-i|A|^{2} A-\mu A+\gamma \bar{A},
$$

where $\mu=\alpha\left(h+\frac{1}{2}\right)-g_{0}$ stands for linear dissipation, and $\gamma=\left(b / 2 \omega_{0}\right) g_{1} \approx \alpha g_{1} /[4 \sqrt{h(1+h)}]$ represents the forcing amplitude. In the conservative limit $\mu=\gamma=0$, the PDNLS equation becomes the well-known nonlinear Schrödinger equation, which describes the amplitude of Hamiltonian oscillations. In the dissipative case $\mu, \gamma>0$, the balance between injection and dissipation generates attracting states, and permanent behaviors such as self-sustained precessions. Note that in this representation the solutions can be obtained analytically (see Ref. [12] and references therein). Moreover, the bifurcation diagram of the PDNLS model is the same as shown in the left panel of Fig. 2.

The amplitude Eq. (8) can be derived using symmetry arguments. When there is no injection or dissipation, the magnetization dynamics are invariant under time reversion $t \rightarrow-t$ and temporal translation $t \rightarrow t+\Delta t$. Hence, the amplitude $A$ must satisfy $(t, A) \rightarrow(-t, \bar{A})$ and $(t, A) \rightarrow\left(t+\Delta t, A e^{-i \Delta}\right)$. Then, the amplitude equation at dominant order reads $\dot{A}=$ $i c_{1} A+i c_{2}|A|^{2} A$, where $c_{1}$ and $c_{2}$ are real constants. In the presence of dissipation and parametric forcing, the time inversion and translation symmetries are broken, respectively. Taking into account these effects, the amplitude equation fulfills $\dot{A}=i c_{1} A+i c_{2}|A|^{2} A+c_{3} A+c_{4} \bar{A}$, where the extra terms depend on damping and driving forces. Therefore, the dynamical behaviors exhibited by PDSTNO belong to the universality class of parametrically driven systems [21].

It is worth noting that all the parameters of the PDNLS equation are controlled by the electric current. Furthermore, under the scaling $\gamma \sim \mu \sim v \sim|A|^{2} \sim d / d t \ll 1$ in Eq. (8), the coupling between the time-varying injection $\gamma$ and the oscillation envelope $A(t)$ is of the same order as the other physical effects. This makes the parametric forcing an efficient mechanism for controlling the magnetization dynamics.

\section{PARAMETRICALLY INDUCED SPATIAL TEXTURES}

Physical systems with a parametric forcing exhibit a large variety of spatially self-organized states [21]. Hence, the feasibility of PDSTNO devices to generate parametric instabilities offers a great advantage for pattern forming studies in the nanoscale domain with respect to other magnetic systems. 
In the next subsections we study the inhomogeneous magnetic states $\mathbf{m}=\mathbf{m}(\mathbf{r}, t)$ induced by the alternating current. Using a simplified magnetic model and the amplitude equation approach we predict the existence of subharmonic standing waves, or Faraday-type waves, and solitons. These predictions are in agreement with our micromagnetic simulations.

\section{A. Analytic approach to nonuniform dynamics}

A minimal model can be obtained neglecting the effects of the borders and approximating the demagnetizing energy by its leading-order contribution in terms of a shape anisotropy. The last approximation is valid when the magnetization has small deformations with respect to the uniform state and the free layer thickness is small compared with lateral sizes.

For spatially varying magnetizations $\mathbf{m}(\mathbf{r}, t)$, the LLGS model and the effective field $\mathbf{h}_{\text {eff }}$ become [35]

$$
\begin{aligned}
\frac{\partial \mathbf{m}}{\partial t} & =-\mathbf{m} \times \mathbf{h}_{\mathrm{eff}}+\alpha \mathbf{m} \times \frac{\partial \mathbf{m}}{\partial t}+g \mathbf{m} \times(\mathbf{m} \times \hat{x}), \\
\mathbf{h}_{\mathrm{eff}} & =\left(h_{0}+\beta m_{x}\right) \hat{x}-m_{z} \hat{z}+\nabla^{2} \mathbf{m},
\end{aligned}
$$

where the last term of $\mathbf{h}_{\text {eff }}$ accounts for the ferromagnetic exchange, and it penalizes inhomogeneities in the magnetization. The spatial operator $\nabla=\hat{y} \partial_{y}+\hat{z} \partial_{z}$ is expressed in the unit of the exchange length $l_{\mathrm{ex}}=\sqrt{2 A_{\text {ex }} /\left(\mu_{0} M_{s}^{2}\right)}$ where $A_{\mathrm{ex}}$ is the exchange stiffness constant.

In this scenario the oscillation envelope $A(\mathbf{r}, t)$ can be described using the following ansatz in Eq. (9a):

$$
m_{y}=m_{y, 0} e^{i\left(\omega_{0}+v\right) t} A(\mathbf{r}, t)+\text { c.c. }+W_{y},
$$

After straightforward calculations, in a similar way to the previous section, we obtain the PDNLS equation:

$$
\frac{\partial A}{\partial t}=-i \nu A-i|A|^{2} A-\mu A+\gamma \bar{A}-i \nabla^{\prime 2} A,
$$

where the last term of Eq. (11) accounts for the dispersion with $\nabla^{\prime}=\left[(2 h+1) /\left(2 \omega_{0}\right)\right]^{1 / 2} \nabla$. The sign of dispersion and nonlinearity renders this equation to a focusing type [36]. Notice that the Laplacian term is the leading-order spatial coupling that accounts for spatial reflection $\mathbf{r} \rightarrow-\mathbf{r}$ and translation $\mathbf{r} \rightarrow \mathbf{r}+\Delta \mathbf{r}$ symmetries.

The amplitude equation (11) is the prototype model describing the envelope of coupled nonlinear oscillators. Furthermore the PDNLS equation allows us to relate the magnetization dynamics of PDSTNO into the same phenomenology of a wide spectrum of physical systems that ranges from nonlinear optical systems to fluids dynamics under driving forces.

The amplitude Eq. (11) is characterized by exhibiting Faraday-type waves and dissipative solitons [21]. The origin of Faraday-type waves is a spatial instability of the parallel state $A=0$. As a result of this destabilization, the noise-induced perturbations are exponentially amplified in time. The growth rate $\sigma$ of the small perturbations $A \sim e^{\sigma t+i \mathbf{k} \cdot \mathbf{r}}$ is obtained linearizing Eq. (11) around zero:

$$
\sigma=-\mu \pm \sqrt{\gamma^{2}-\left(|\mathbf{k}|^{2}-v\right)^{2}},
$$

where $\mathbf{k}$ is the wave vector of the modes in which perturbations are decomposed. For alternating currents above the critical value $\gamma_{c}=\mu$, the modes with wave number $|\mathbf{k}|=\sqrt{v}$ grow and destabilize the uniform configuration. The zone where
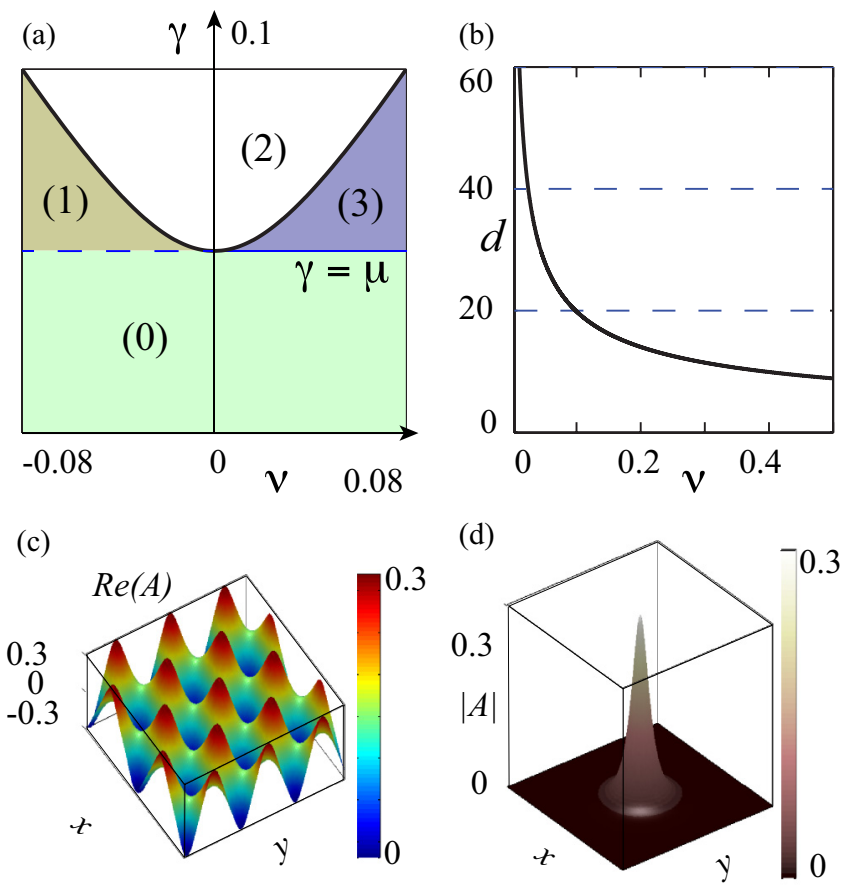

FIG. 3. (Color online) Phases diagram of the PDNLS model, Eq. (11). (a) Detuning-injection plane; in zones (1) and (3) solitons and standing waves exist. Region (2) is known as the Arnold tongue; in this zone the parallel state $A=0$ becomes unstable, and then solitons also are unstable. Standing waves are the only steady states observed in this region. In zone (3) standing waves emerge by supercritical bifurcation. (b) Wavelength for Faraday-type waves as a function of detuning. (c) Typical Faraday-type waves obtained for $\gamma=0.055, \mu=0.05$, and $v=0.075$. (d) Dissipative soliton, parameters $\gamma=0.0505, \mu=0.05$, and $v=-0.015$.

Faraday-type waves exist is marked as (3) in Fig. 3(a). It is worth noting that the necessary conditions $\gamma>\mu$ and $\nu>0$ to observe Faraday-type waves, as well as their wavelength $d=2 \pi l_{\mathrm{ex}} / \sqrt{\nu}$, are completely controlled by the electric current parameters $\left\{g_{1}, g_{0}, \omega\right\}$, or equivalently the PDNLS parameters $\left\{\gamma\left(g_{1}\right), \mu\left(g_{0}\right), v(\omega)\right\}$. Figure 3(b) shows the typical wavelengths, in units of the exchange length, as a function of the detuning parameter. As this figure illustrates, for positive detuning, typical wavelengths are of order $d \sim 15 l_{\mathrm{ex}}$, which is about $d_{\text {Co }}=52(\mathrm{~nm})$ and $d_{\text {Py }}=85(\mathrm{~nm})$ for cobalt and Permalloy, respectively. Smaller wavelengths can be obtained by increasing the forcing frequency $\omega$.

At the onset of the spatial instability and after transients, the standing wave is composed of one or a few Fourier modes, compatible with boundary conditions and having a wave number close to the critical value $\left|\mathbf{k}_{\mathbf{c}}\right| \approx \sqrt{v}$. Figure $3(\mathrm{c})$ shows a typical standing wave of Eq. (11). In general, the number of such modes, as well as the orientation of their wave vectors, depend on the nonlinear saturation mechanisms of the particular problem, material defects, and borders [42].

This type of instability was studied in spin valves for the case of constant external field and direct spin-polarized current [11,12]. A PDNLS model was derived [12] using an appropriate time-independent change of variables of the form $A \sim\left(m_{y}+i m_{z}\right) /\left(1+m_{x}\right)$. Even if the system under study in Refs. [11,12] does not have parametric forcing, the 
magnetization obeys a PDNLS equation, and textures usually found in parametrically driven systems emerge [12]. This correspondence between parametric systems and spin valves with direct current was called parametric equivalence. In the parametric equivalence, the origin of the parametric injection (term $\gamma \bar{A}$ ) is related to anisotropy effects. The direct spinpolarized current was responsible for the dissipation term $\mu A$. It is worth noting that in the parametric equivalence most of the magnetic textures are static and periodic in space. In opposition, when an alternating current is applied, the magnetization oscillates both in space and time $m_{y} \sim e^{i \omega_{0} t} \cos (i \mathbf{k} \cdot \mathbf{r})+$ c.c. Since the anisotropies are fixed in usual setups, the only control parameters in the parametric equivalent system are the detuning (external magnetic field) and dissipation (the constant electric current). In our present case, the amplitude $g_{1}$ and frequency $\omega$ of the alternating current and the direct current $g_{0}$ are the control parameters associated to the parametric injection $\gamma$, detuning $\nu$, and dissipation $\mu$, respectively. In brief, the use of an alternating current permits a more adequate control of the parametric behaviors exhibited by the PDNLS model.

Dissipative solitons are another prominent example of spatial self-organization in macroscopic nonlinear systems. They can be described by particlelike attributes, such as position and width [43].

The parametric forcing induces dissipative solitons that connect asymptotically the quiescent state $A=0$. Figure 3(d) shows a soliton state, obtained from Eq. (11). There is no analytic expression for this solution in PDNLS model, nevertheless they can be approximated using the variational method $[44,45]$ by

$$
A_{\text {soliton }}(\mathbf{r})=a_{0} \sqrt{\lambda} e^{i \phi_{0}} \operatorname{sech}\left(b_{0} \sqrt{\lambda}|\mathbf{r}|\right),
$$

where $\lambda \equiv-v+\sqrt{\gamma^{2}-\mu^{2}}, \cos \left(2 \phi_{0}\right)=\mu / \gamma, a_{0}=2.166$, and $b_{0}=0.933$. This approximation gives a characteristic amplitude $a_{0} \lambda^{1 / 2}$, and a characteristic width $b_{0}^{-1} \lambda^{-1 / 2}$. In a similar way to the Faraday-type waves wavelengths, the electric current parameters $\left\{g_{1}, g_{0}, \omega\right\}$ control the soliton typical lengths.

We can see from formula (13) that, at dominant order, the soliton decays exponentially to the parallel state $A \sim$ $e^{-\sqrt{\lambda}|\mathbf{r}|} \rightarrow 0$ far from the center of the soliton, that is, $|\mathbf{r}| \gg$ $\lambda^{-1 / 2}$. Since, soliton tails connect the homogeneous state, solitons are observed only where the parallel state is stable. These zones are labeled as (0) and (1) in Fig. 3. Hence, solitons exist for negative detuning and injection amplitudes in the range $\mu<\gamma<\sqrt{\mu^{2}+v^{2}}$, which corresponds to zone (1) in Fig. 3(a).

\section{B. Micromagnetic simulation of solitons and Faraday-type waves}

To investigate the robustness of the observed textures, and to illustrate the parametric instability of spin valves, we model a $500 \times 500 \times 2-\mathrm{nm}^{3}$ Permalloy free layer sample $\left(M_{s}=800 \mathrm{kA} / \mathrm{m}, K=14.5 \mathrm{~kJ} / \mathrm{m}^{3}\right)$ with a discretization of $127 \times 127 \times 1$ cells with a Neumann boundary condition. We use an adaptive step size fifth-order Runge-Kutta scheme. The effective field includes the full demagnetization term [46]

$$
\mathbf{h}_{\mathrm{eff}}=\left(h_{0}+\beta m_{x}\right) \hat{x}-\nabla \Phi+\nabla^{2} \mathbf{m}
$$

where the magnetic potential $\Phi$ is

$$
\Phi(\mathbf{r})=\frac{1}{4 \pi} \int_{V} \frac{\rho_{m}\left(\mathbf{r}^{\prime}\right)}{\left|\mathbf{r}-\mathbf{r}^{\prime}\right|} d^{3} r^{\prime}+\frac{1}{4 \pi} \int_{\partial V} \frac{\sigma_{m}\left(\mathbf{r}^{\prime}\right)}{\left|\mathbf{r}-\mathbf{r}^{\prime}\right|} d^{2} r^{\prime},
$$

and the charge densities in the bulk $\rho_{m}=-\nabla \cdot \mathbf{m}$ and at the surface $\sigma_{m}=\hat{n} \cdot \mathbf{m}$ are the magnetic analog of electric charge densities. The density $\rho_{m}$ is induced by nonuniformly magnetic distributions, while $\sigma_{m}$ appears if the magnetization points towards the outward normal $\hat{n}$ at the sample borders [46].

We obtain the demagnetization field using the discrete convolution [47] between the magnetization and the demagnetization tensor. The demagnetization tensor components are generated by the function included in the OOMMF code [48,49]. We use an external field $H=0.2 M_{s}$ and a direct current density $J_{\mathrm{dc}}=0.16 \mathrm{~A} / \mu \mathrm{m}^{2}$; for a polarization factor $\mathcal{P}=0.3$ we obtain the following reduced parameters: $h=$ $0.2, \beta=0.03$, and $g_{0}=0.01$. That is, the critical value of the radio-frequency current for the parametric instability is $g_{1}=0.034\left(J_{\mathrm{ac}}=0.55 \mathrm{~A} / \mu \mathrm{m}^{2}\right)$. In this configuration the natural frequency is $\omega_{0}=0.53\left(\Omega_{0}=14.85 \mathrm{GHz}\right)$ and for the detunings $v_{1}=-0.004\left(112 \mathrm{MHz}\right.$ below $\left.\Omega_{0}\right)$ and $\nu_{2}=0.075$ $\left(2 \mathrm{GHz}\right.$ above $\left.\Omega_{0}\right)$ a localized state and Faraday-type waves are expected, respectively. Fixing the effective alternate current at $g_{1}=0.2\left(3.22 \mathrm{~A} / \mu \mathrm{m}^{2}\right)$ we obtain different spatial structures that can be seen from Figs. 4(a) and 4(b) for $v_{1}$ and $v_{2}$, respectively. Hence, solitons and Faraday-type waves persist when the demagnetizing effects are included [50]. Since the magnetization is approximately in the $x$ axis, the main demagnetization contribution at the in-plane borders is a surface magnetic charge of the form $\sigma_{m}= \pm \mathbf{m} \cdot \hat{x}$. Figure 5 shows the typical deviations induced by the effect of magnetic charges.

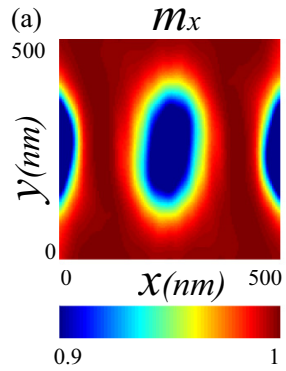

(b)
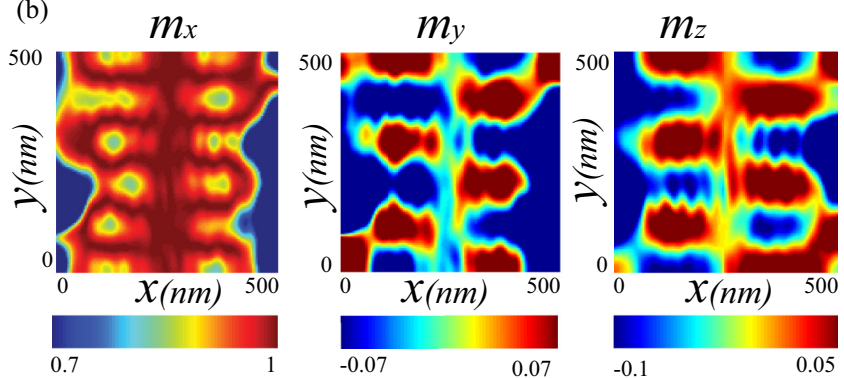

FIG. 4. (Color online) Parametrically induced textures. Dissipative solitons (a) and Faraday-type waves (b) obtained from simulations of the LLG equation with the effective field (14) for a $500 \times 500 \times 2-\mathrm{nm}^{3}$ Permalloy sample with $v=-0.004$ and 0.075 , respectively. The Gilbert damping parameter is fixed at $\alpha=0.014$. 

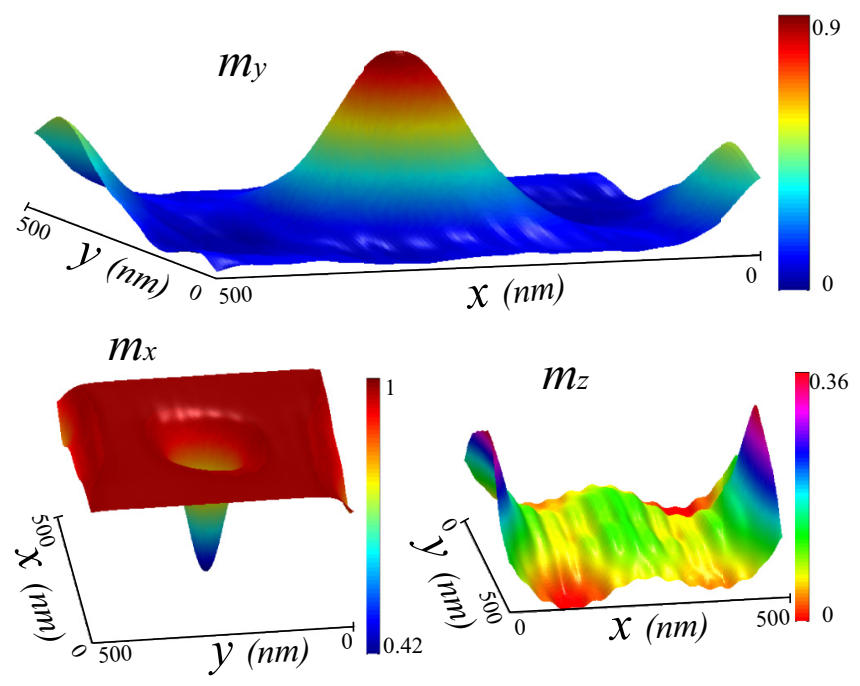

FIG. 5. (Color online) Dissipative solitons in parametrically driven spin valves obtained from Eq. (9b) using the effective field (14). Solitons persist when the sample borders and the full dipolar field are considered.

\section{CONCLUSIONS AND REMARKS}

We have shown that a spin-polarized electric current, with both constant and oscillatory components, induces parametric instabilities in spin valves. In the case of macrospin approximation, additional equilibria and precessional states emerge. This scenario changes when the spatial variations of the magnetization are considered. In particular, our analysis shows that the free magnetic layer exhibits a wide class of robust self-organization phenomena observed in driven systems, such as dissipative solitons and Faraday-type waves. These states are robust when additional effects, such as the dipolar field or the sample borders, are considered. Hence, the use of alternating spin-polarized currents opens the possibility to control transitions from the parallel state to uniform precessions or dissipative solitons and Faraday-type waves. These behaviors could open novel and fresh functionality to spin-valve devices.

\section{ACKNOWLEDGMENTS}

We thank H. Pleiner for fruitful discussions. The authors acknowledge support from Fondo Nacional de Desarrollo Científico y Tecnológico Grants No. 1150507, No. 1120764, and No. 1150072; Basal Program Center for Development of Nanoscience and Nanotechnology (CEDENNA); Engineering and Physical Sciences Research Council Grant No. EP/L002922/1; Anillo de Ciencia y Tecnonología ACT Grant No. 1117; UTA Project No. 8750-12; and Becas Conicyt 2012 Contract No. 21120878. S.C. acknowledges the financial support of Ministry of Higher Education and Research, NordPas de Calais Regional Council and ERDF through the CPER 2007-2013, as well as by the ANR LABEX CEMPI project (No. ANR-11-LABX-0007).
[1] S. I. Kiselev, J. C. Sankey, I. N. Krivorotov, N. C. Emley, R. J. Schoelkopf, R. A. Buhrman, and D. C. Ralph, Microwave oscillations of a nanomagnet driven by a spin-polarized current, Nature (London) 425, 380 (2003); D. C. Ralph and M. D. Stiles, Spin transfer torques, J. Magn. Magn. Mater. 320, 1190 (2008); G. Bertotti, C. Serpico, I. D. Mayergoyz, A. Magni, M. d'Aquino, and R. Bonin, Magnetization switching and microwave oscillations in nanomagnets driven by spin-polarized currents, Phys. Rev. Lett. 94, 127206 (2005), and references therein.

[2] B. Georges, J. Grollier, M. Darques, V. Cros, C. Deranlot, B. Marcilhac, G. Faini, and A. Fert, Coupling efficiency for phase locking of a spin transfer nano-oscillator to a microwave current, Phys. Rev. Lett. 101, 017201 (2008).

[3] Z. Yang, S. Zhang, and Y. C. Li, Chaotic dynamics of spin-valve oscillators, Phys. Rev. Lett. 99, 134101 (2007).

[4] D. Li, Y. Zhou, C. Zhou, and B. Hu, Fractional locking of spintorque oscillator by injected ac current, Phys. Rev. B 83, 174424 (2011), and references therein.

[5] J. C. Slonczewski, Current-driven excitation of magnetic multilayers, J. Magn. Magn. Mater. 159, L1 (1996).

[6] L. Berger, Emission of spin waves by a magnetic multilayer traversed by a current, Phys. Rev. B 54, 9353 (1996).

[7] Z. Li, Y. C. Li, and S. Zhang, Dynamic magnetization states of a spin valve in the presence of $\mathrm{dc}$ and ac currents: Synchronization, modification, and chaos, Phys. Rev. B 74, 054417 (2006).

[8] M. d'Aquino, C. Serpico, R. Bonin, G. Bertotti, and I. D. Mayergoyz, Stochastic resonance in noise-induced transitions between self-oscillations and equilibria in spin-valve nanomagnets, Phys. Rev. B 84, 214415 (2011), and references therein.

[9] K. J. Lee, A. Deac, O. Redon, J. P. Nozieres, and B. Dieny, Excitations of incoherent spin-waves due to spin-transfer torque, Nat. Mater. 3, 877 (2004).

[10] X. W. Yu, V. S. Pribiag, Y. Acremann, A. A. Tulapurkar, T. Tyliszczak, K. W. Chou, B. Bräuer, Z. P. Li, O. J. Lee, P. G. Gowtham, D. C. Ralph, R. A. Buhrman, and J. Stöhr, Images of a spin-torque-driven magnetic nano-oscillator, Phys. Rev. Lett. 106, 167202 (2011).

[11] A. O. León, M. G. Clerc, and S. Coulibaly, Dissipative structures induced by spin-transfer torques in nanopillars, Phys. Rev. E 89, 022908 (2014).

[12] A. O. León and M. G. Clerc, Spin-transfer-driven nanooscillators are equivalent to parametric resonators, Phys. Rev. B 91, 014411 (2015).

[13] S.-B. Choe, Y. Acremann, A. Scholl, A. Bauer, A. Doran, J. Stöhr, and H. A. Padmore, Vortex core driven magnetization dynamics, Science 304, 420 (2004).

[14] K. Yu. Guslienko, Magnetic vortex state stability, reversal and dynamics in restricted geometries, J. Nanosci. Nanotechnol. 8, 2745 (2008).

[15] Q. Mistral, M. van Kampen, G. Hrkac, Joo-Von Kim, T. Devolder, P. Crozat, C. Chappert, L. Lagae, and T. Schrefl, Current-driven vortex oscillations in metallic nanocontacts, Phys. Rev. Lett. 100, 257201 (2008).

[16] A. Slavin and V. Tiberkevich, Spin wave mode excited by spinpolarized current in a magnetic nanocontact is a standing selflocalized wave bullet, Phys. Rev. Lett. 95, 237201 (2005). 
[17] S. Bonetti, V. Tiberkevich, G. Consolo, G. Finocchio, P. Muduli, F. Mancoff, A. Slavin, and J. Akerman, Experimental evidence of self-localized and propagating spin wave modes in obliquely magnetized current-driven nanocontacts, Phys. Rev. Lett. 105, 217204 (2010).

[18] R. K. Dumas, E. Iacocca, S. Bonetti, S. R. Sani, S. M. Mohseni, A. Eklund, J. Persson, O. Heinonen, and J. Akerman, Spinwave-mode coexistence on the nanoscale: A consequence of the oersted-field-induced asymmetric energy landscape, Phys. Rev. Lett. 110, 257202 (2013).

[19] S. M. Mohseni et al., Spin torque generated magnetic droplet solitons, Science 339, 1295 (2013).

[20] M. A. Hoefer, T. J. Silva, and M. W. Keller, Theory for a dissipative droplet soliton excited by a spin torque nanocontact, Phys. Rev. B 82, 054432 (2010).

[21] M. G. Clerc, S. Coulibaly, and D. Laroze, Localized states beyond the asymptotic parametrically driven amplitude equation, Phys. Rev. E 77, 056209 (2008); Non-variational Ising-Bloch transition in parametrically driven systems, Int. J. Bifurcation Chaos 19, 2717 (2009); Parametrically driven instabilities in quasi-reversal systems, 19, 3525 (2009); Localized states and non-variational Ising-Bloch transition of a parametrically driven easy-plane ferromagnetic wire, Physica D 239, 72 (2010); Localized waves in a parametrically driven magnetic nanowire, Europhys. Lett. 97, 30006 (2012).

[22] D. Urzagasti, D. Laroze, M. G. Clerc, S. Coulibaly, and H. Pleiner, Two-soliton precession state in a parametrically driven magnetic wire, J. Appl. Phys. 111, 07D111 (2012).

[23] D. Urzagasti, A. Aramayo, and D. Laroze, Soliton-Antisoliton interaction in a parametrically driven easy-plane magnetic wire, Phys. Lett. A 378, 2614 (2014).

[24] D. Urzagasti, D. Laroze, and H. Pleiner, Localized chaotic patterns in weakly dissipative systems, Eur. Phys. J. Special Topics 223, 141 (2014)

[25] A. O. León, M. G. Clerc, and S. Coulibaly, Traveling pulse on a periodic background in parametrically driven systems, Phys. Rev. E 91, 050901 (2015).

[26] M. Clerc, P. Coullet, and E. Tirapegui, Lorenz bifurcation: Instabilities in quasireversible systems, Phys. Rev. Lett. 83, 3820 (1999); The stationary instability in quasi-reversible systems and the Lorenz pendulum, Int. J. Bifurcation Chaos 11, 591 (2001).

[27] S. Fauve, in Hydrodynamics and Nonlinear Instabilities, edited by C. Godrèche and P. Manneville (Cambridge University Press, Cambridge, 1998), Chap. 4 and references therein.

[28] C. Elphick, E. Tirapegui, M. Brachet, P. Coullet, and G. Iooss, A simple global characterization for normal forms of singular vector fields, Physica D 29, 95 (1987).

[29] I. V. Barashenkov, M. M. Bogdan, and V. I. Korobov, Stability diagram of the phase-locked solitons in the parametrically driven, damped nonlinear Schrödinger equation, Europhys. Lett. 15, 113 (1991).

[30] M. G. Clerc, S. Coulibaly, M. A. Garcia-Ñustes, and Y. Zárate, Dissipative localized states with shieldlike phase structure, Phys. Rev. Lett. 107, 254102 (2011).
[31] S. Fauve and O. Thual, Solitary waves generated by subcritical instabilities in dissipative systems, Phys. Rev. Lett. 64, 282 (1990).

[32] M. Faraday, On a peculiar class of acoustical figures; and on certain forms assumed by groups of particles upon vibrating elastic surfaces, Philos. Trans. R. Soc. London 121, 299 (1831).

[33] C. Wang, H. Seinige, and M. Tsoi, Current-driven parametric resonance in magnetic multilayers, J. Phys. D 46, 285001 (2013).

[34] P. Bortolotti et al., Parametric excitation of magnetic vortex gyrations in spin-torque nano-oscillators, Phys. Rev. B 88, 174417 (2013).

[35] Z. Li and S. Zhang, Magnetization dynamics with a spin-transfer torque, Phys. Rev. B 68, 024404 (2003); J. Z. Sun, Spin-current interaction with a monodomain magnetic body: A model study, ibid. 62, 570 (2000); X. Waintal, E. B. Myers, P. W. Brouwer, and D. C. Ralph, Role of spin-dependent interface scattering in generating current-induced torques in magnetic multilayers, ibid. 62, 12317 (2000); M. D. Stiles and A. Zangwill, Anatomy of spin-transfer torque, ibid. 66, 014407 (2002).

[36] Y. S. Kivshar and G. P. Agrawal, Optical Solitons: From Fibers to Photonic Crystals (Academic Press, San Diego, 2003).

[37] J. Xiao, A. Zangwill, and M. D. Stiles, Macrospin models of spin transfer dynamics, Phys. Rev. B 72, 014446 (2005).

[38] L. Cesari, Asymptotic Behaviour and Stability Problems in Ordinary Differential Equations (Springer, New York, 1962).

[39] E. I. Butikov, Subharmonic resonances of the parametrically driven pendulum, J. Phys. A 35, 6209 (2002).

[40] C. M. Bender and S. A. Orzag, Advanced Mathematical Methods for Scientists and Engineers (McGraw-Hill, New York, 1978).

[41] V. I. Arnold, Geometrical Methods in the Theory of Ordinary Differential Equations (Springer-Verlag, New York, 1998).

[42] R. B. Hoyle, Pattern Formation: An Introduction to Methods (Cambridge University Press, Cambridge, 2006).

[43] M. Remoissenet, Waves Called Solitons (Springer, Berlin, 1999).

[44] D. Anderson, M. Bonnedal, and M. Lisak, Self-trapped cylindrical laser beams, Phys. Fluids 22, 1838 (1979).

[45] M. G. Clerc, S. Coulibaly, and D. Laroze, Interaction law of 2D localized precession states, Europhys. Lett. 90, 38005 (2010).

[46] J. M. D. Coey, Magnetism and Magnetic Materials (Cambridge University Press, Cambridge, 2010).

[47] N. Hayashi, K. Saito, and Y. Nakatani, Calculation of demagnetizing field distribution based on fast Fourier transform of convolution, Jpn. J. Appl. Phys. 35, 6065 (1996).

[48] A. J. Newell, W. Williams, and D. J. Dunlop, A generalization of the demagnetizing tensor for nonuniform magnetization, J. Geophys. Res. Solid Earth 98, 9551 (1993).

[49] M. Donahue and D. Porter, The Object Oriented MicroMagnetic Framework (OOMMF project at ITL/NIST), http://math.nist.gov/oommf/.

[50] See Supplemental Material at http://link.aps.org/supplemental/ 10.1103/PhysRevB.91.224426 for a movie that shows the spatiotemporal dynamics of these spatial structures. 